\title{
A study on Fuzzy Cognitive Map structures for Medi- cal Decision Support Systems
}

\author{
EvangeliaBourgani ${ }^{1}$,Chrysostomos D. Stylios ${ }^{1}$,Voula C. Georgopoulos ${ }^{2}$ and George Manis ${ }^{3}$ \\ ${ }^{1}$ Dept. of Informatics and Telecommunications Technology, TEI of Epirus, Artas, Greece (email: \\ ebourgani@gmail.com; stylios@teiep.gr) \\ ${ }^{2}$ School of Health and Welfare Professions, TEI of Western Greece, Patras, Greece (email: voula@teipat.gr ) \\ ${ }^{3}$ Dept. of Computer Science \& Engineering, University of Ioannina, Ioannina, Greece (email manis@cs.uoi.gr)
}

\begin{abstract}
This study examines and compares different Fuzzy Cognitive Map structures that researchers have proposed for developing Medical Decision Support Systems. Fuzzy Cognitive Maps are a soft computing technique that have gained a good reputation in the last decade and have been used successfully in different medical fields for decision making, diagnosis and classification.
\end{abstract}

Keywords: Fuzzy Cognitive Maps, Medical Decision Support Systems

\section{Introduction}

Medical Decision Making is a complex procedure, taking into consideration a variety of factors and it infers a diagnosis/decision. Medical Decision Support Systems (MDSS) are planned to support human decision making successfully evaluating information from different sources, combining it to support clinicians' decisions concerning diagnosis, therapy planning and monitoring of the disease and treatment processes. Medical Decision Systems have to consider a high amount of data and information from interdisciplinary sources (patient's records and information, doctors' physical examination and evaluation, laboratory tests, ultrasound and other medical devices etc) and, in addition, information may be vague, missing or not available. Thus, Medical systems are complex systems involving inexact, uncertain, imprecise and ambiguous information [1]. These systems can provide assistance in crucial clinical judgments, particularly for inexperienced medical professionals.

Fuzzy Cognitive Maps (FCMs) are a soft computing technique used for causal knowledge acquisition and supporting causal knowledge reasoning process. The FCM modeling approach resembles human reasoning; it relies on the human expert knowledge for a domain, making associations along generalized relationships between domain descriptors, concepts and conclusions. It models any real world system as a collection of concepts and causal relation between concepts.

This work presents a study on MDSS based on FCMs and their variants. More specifically, it reports the FCM structures that have been developed for medical appli- cations, their methodologies for MDSS and compares them.

\section{Fuzzy Cognitive Maps}

An FCM is illustrated as a causal graphical representation consisting of interrelated concepts. It was introduced [2]as an extension to Cognitive Maps [3].The general graphical illustration of FCM is a signed, weighted graph with feedback. Nodes of the graph are concepts, which correspond to variables, states, factors and other characteristics that are used in the model (that describe the behavior of the system). Causal relationships interconnect the FCM concepts as illustrated in Fig.1.

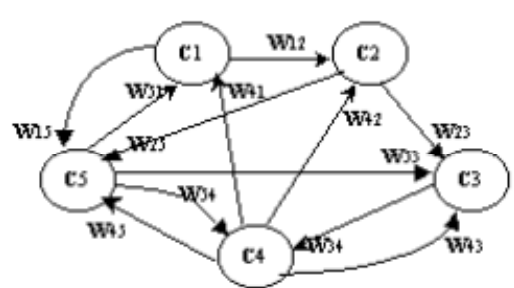

Fig. 1: The fuzzy cognitive map model

An FCM is developed by experts who describe each interconnection with a fuzzy rule, which infers a linguistic variable that describes the relationship between the two concepts according to every one expert and determines the grade of causality between the two concepts. The inferred fuzzy weights are aggregated and deffuzified giving an initial numerical weight in the interval $[-1,1]$. One significant weakness of FCMs is the critical dependence on the experts' opinions; another essential one is the potential convergence to undesired steady states. The use of learning procedures can enhance FCMs and increase their efficiency and robustness. The desired steady state is characterized by values of the FCM's output concepts that are accepted by the experts.

FCMs develop a behavioral model of the system that exploits the experience and expert knowledge. This FCM's applicability to model complex system has been successfully used in many different application areas.

\section{Medical Decision Support Systems}

Medical Decision Support Systems (MDSS) follow the general nature of medical interventions: predict (predictive machine), prevent (preventive medicine), heal (curative medicine), or at least comfort (medical assis- 
tance) [4]. For this goal, a patient's particular situation must be considered (diagnosis or prognosis) and possible strategies be evaluated. Medical Decision Systems are complex systems consisting of irrelevant and relevant subsystems and elements, taking into consideration many factors that may be complementary, contradictory, and competitive; these factors influence each other and determine the overall diagnosis with a different degree. A MDSS System usually extracts causal knowledge from the appropriate medical domain, build a causal knowledge base, and make inference with it. FCMs can give this opportunity.

MDSS have been used for medical decision making, reasoning, differential diagnosis, prediction and/or treatment purposes.

\section{FCM for Medical Decision Support Systems}

FCMs have been used successfully to develop Decision Support Systems (DSS). The major advantage of FCMs is that they can handle even incomplete or conflicting information. This is essential, especially in medicine where experts should take many factors under consideration before they can reach a decision. Different FCM structures and learning methods have been developed for medical decision support systems. Fig.2 illustrates FCM structures that have been applied to Medical Support Systems.

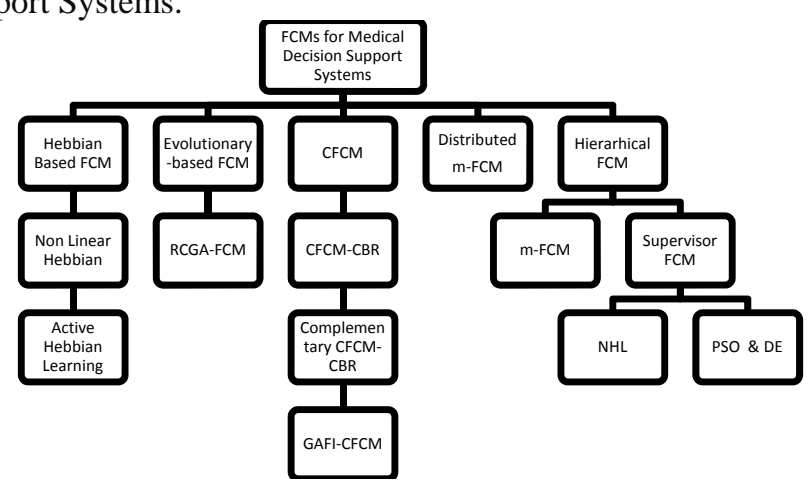

Fig. 2: FCM structures for MDSS

\subsection{FCM based on HebbianLearning Methods}

FCMs have been enhanced with learning algorithms. Adaptation and learning methodologies based on ANN are used to adapt the FCM model and adjust its weights. Kosko and Dickerson[5] presented the Differential Hebbian Learning (DHL) to train FCMs, but without mathematical formulation and implementation in any problem. An improved version of DHL learning was introduced by Huerga[6], called Balanced Differential Algorithm (BDA). However, BDA works only on binary FCMs. To overcome the limitations of using the existing learning methods from Neural Networks domains into FCMs, two methods have been proposed: the non Linear Hebbian Learning [7] and Active Hebbian Learning algorithms [8].

\subsubsection{FCM with Non Linear Hebbian Learning}

The Non Linear Hebbian Learning (NHL) method is a semi-automated approach that requires initial human intervention [7]. This algorithm is based on the Hebbian theory, but it uses a nonlinear extension to the basic Hebbian Rule[9] by introducing modified weight update formula. Experts are required to suggest nodes that are directly connected and only these edges are updated during the learning.NHL algorithm is based on the premise that all the concepts in FCM model trigger synchronously at each iteration step.

The advantage of NHL is that it updates only those weights that experts determined, that is, the non-zero weights. The weight values of the FCM are updated synchronously and they have fixed values for the entire learning process (which retains the structure of obtaining model). Thus, the NHL does not assign new interconnections and the zero weights do not change. However, this learning method requires human intervention before the learning process. The construction of FCMs is based on experts that make them subjective. Besides, the learning method may conclude to a non-convergent output value, which means rescheduling the treatment planning by experts. The NHL algorithm does not require historical data and it relies on expert-derived initial map and a set of terminal conditions.

NHL has been applied to radiation therapy for decision making about the final dose of radiation. Papageorgiou et al.[7] proposed the Clinical Treatment Simulation Tool for Decision making in radiotherapy (CTST-FCM) model which is based on NHL algorithm. The CTST-FCM tool is efficient and useful especially for the case of complex radiotherapy treatment, as it can give information about the influence of other characteristics (such as the volume of healthy tissue or organs that can absorb radiation apart from the tumor).FCMNHL has been also used for tumor grading tool in order to achieve tumor malignancy classification.

However, there are some factors, which change their values easily and they should be taken under consideration during the final decision-making process. For this reason, a better solution would be the designing of a higher level with new key-concept. This led to the construction of a supervisor-FCM which is referred to as the hierarchical architecture of FCM. The proposed two-level hierarchical architecture combines two systems achieving better results. Supervisor-FCM supervises the radiotherapy process and 'decides' the acceptance or not of the treatment therapy.

\subsubsection{FCM with Active Hebbian Learning (AHL)}

For constructing FCM models with dynamic behavior, Active Hebbian Learning (AHL), another unsupervised learning method based on Hebbian theory is used. AHL introduces the determination of the sequence of activation concepts and improves the accuracy. The algorithm adjusts and modifies the weights of FCMs improving the FCM's efficiency and adaptability. The AHL adds causal links between all the concepts so as to achieve the desired behavior of the system. Thus, all the weights are updated at the end of learning cycle and new causal interconnections are assigned.

The AHL algorithm takes into consideration the experts' knowledge and experience for the initial values of the weights, which are derived from the summation 
of experts' opinions [8]. Besides, it supposes that there is a sequence of activation concepts, which depends on the specific problem's configuration and characteristics. Thus, when experts develop the FCM, they determine the sequence of activation, the steps of activation and the cycle of simulation.

The main advantage of the AHL algorithm is that it can determine new FCM causal links between all the concepts in order to increase classification capabilities of the FCM. In this way the AHL algorithm increases the FCMs' effectiveness, flexibility and robustness, and creates advanced FCMs with dynamic behavior and great modeling abilities where new features can easily be introduced, added or deleted making a continuously evolved model. The AHL Algorithm introduces asynchronous updating of the weights, the Activation and Activated concepts and the calculation of Activation Decision Concepts (ADCs), which are the desired outputs of the system representing the final state.

AHL has been used for classification problems (tumor grading) giving better results compared to FCM, under the same conditions.

Both the AHL and NHL algorithms are problemdependent and they use the initial weight matrix. However, both processes are independent from the initial values of concepts and the system's output concepts manage to converge to the desired equilibrium points with appropriate learning parameters.

FCMs are subjective to experts' opinion. However, experts do not have the same experience or the same background; hence a 'credibility' parameter should be inserted in order to have more reliable results. Besides, neither of these methods takes historical data under consideration.

\subsection{Competitive Fuzzy Cognitive Maps}

Another FCM structure based on human intervention and history data from literature or/and specialists' experience is Competitive FCMs that give solutions to differential decisions/diagnosis. Competitive learning methods for FCMs were successfully applied for medical diagnosis problems because they ensure that only one diagnosis will be reached. Thus, in a differential diagnosis MDSS, where only one diagnosis is inferred, the Competitive FCM (CFCM) can be used [10].

CFCMs are capable on their own to perform a comparison and lead to a decision, based on expert knowledge and experience. In CFCMs there are two main kinds of concepts (diagnoses and factors), which take into account all possible symptoms and factors. Each diagnosis concept represents a single diagnosis, which means that these concepts must be mutually exclusive, because the main intention is to infer always only one diagnosis.

The basic idea of the model is based on the fact that the general diagnosis procedure is a complex process that it has to take under consideration a variety of interrelated factors and functions. Usually, in any real world diagnosis and decision problem, many different factors are taken into consideration and some of these factors are complementary, others are similar and even others are conflicting. All concepts interact with each other until a decision-diagnosis is reached.

In CFCM, the inputs of the system are the factorconcepts (patient data) which are dynamically updated, based on the system interaction. Fig.3 illustrates a FCM, which can handle situations with interconnections and feedback, until its 'competitive' interconnections to reach to a diagnosis.

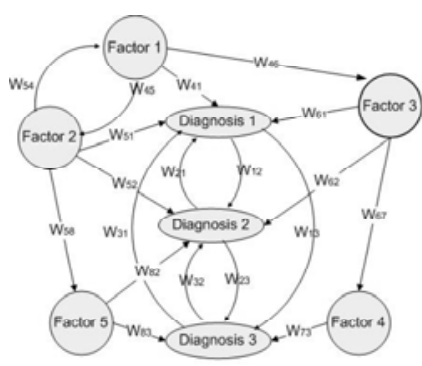

Fig. 3: A CFCM model for Medical Diagnosis

An CFCM has been used for describing differential diagnosis among Specific Language Impairment, Dyslexia and Autism [10]. These disorders have many symptoms in common. This model combines the different factor concepts, which through 'competitiveness' will give the 'winner' concept that constitutes the diagnosis.

The simulation of this model, reaches a decision. However, this may not be a clear one since in certain situations the output concepts have very close values. Thus, the CFCM cannot support the diagnosis of one concept (e.g. Dyslexia), which has the highest value, without additional information.

\subsubsection{Augmented Competitive Fuzzy Cognitive Maps Supplemented with Case Based Reasoning (CBR)}

Augmented FCMs, supplemented with Case Based Reasoning [11], constitute an extension of the CFCMs. They were introduced, in order to ensure that most of the nodes have been activated during the process of taking a decision. As the design of FCMs depends on human experts and learning methods some rare occurrences of medical conditions/symptoms may have been underestimated, keeping many of the nodes inactive. In such circumstances there would be difficulty in taking a decision or leading to an erroneous one. The advantage of CBR-Augmented CFCMs lies on the ability to represent these rare occurrences.

The CBR-Augmented Competitive FCM is capable on its own to perform a comparison and lead to a decision based on expert opinions (structure of CFCM) and previous cases (CBR). The main idea of CBR is the assumption that similar problems usually have similar solutions. The CBR is not called every time (only if a decision cannot be reached, CBR is called)(Fig.4).The detailed process is described in [11].

CFCM-CBR has been applied for diagnosis in speech and language pathology. Specifically, it has developed as a differential diagnostic System for Specific Language Impairment, Autism and Dyslexia [11].It has been applied in comparison with CFCM without CBR and the results showed the advantages of the new system. CFCM-CBR gives a more 'clear' and distinct deci- 
sion of the diagnosis concept, as the one dominant has enough difference from others.

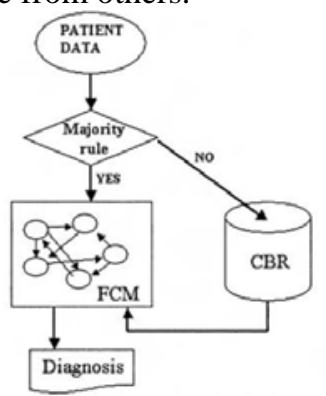

Fig 4: Schematic illustration of CBR augmented FCM

\subsubsection{Complementary CFCM-CBR (CBR enhanced)}

For handling situations where decisions are not clearly distinct the enhanced CBR [12] proposed. The CBRenhanced CFCMs is an extension of competitive FCMs for medical decision support systems by complementing them with case based reasoning methods. It achieves a more distinct and reliable decision, adding optimization criterion.

CBR-enhanced CFCM is capable on its own to perform a comparison and lead to a decision based on expert knowledge and experience (structure of CFCM) and well known tested previous cases (CBR), but this model adds another optimization: the reduction of the search space and time because of the use of a second criterion for the case retrieval and the use of lateral inhibition. Lateral inhibition can enhance differences between different decisions/diagnoses and emphasize boundaries, leading to the single "winner" [13] decision/diagnosis.

The algorithm [12] which used is complementary to the CFCM-CBR's. When CFCM has difficulty to infer a decision with great certainty then the CBR is activated and the minimum difference between input (patient) data and stored is estimated (Fig 5). This comparison takes place in the case-base with decisions/diagnosis corresponding to the highest value of decision/diagnosis concepts of the CFCM (search space and time reduction).The case with the highest similarity is returned to CFCM. It also offers double check of the decision/diagnosis concept and the CFCM is updated. This update becomes according to lateral inhibition, which can inhibit weak connections.Usually, in any diagnosis system there are attributes (factor concepts) whose values are considered critical for each one decision/diagnosis [12].

Thus, the corresponding critical factor concepts are used to inhibit the connections of those factors to the other competitive decision. The advanced medical decision support system based on the complementary usage of competitive FCMs with case-based reasoning (CBR) led to develop the CBR-enhanced CFCM system for medical diagnosis (Fig. 5).

Complementary CFCM-CBR has been applied successfully to model and test two decision support systems: one differential diagnosis problem from the speech pathology area and the other for decision making choices in external beam radiation therapy [12].
As regard the speech pathology, Complementary CFCM-CBR were implemented under the same conditions as the previous CFCMs and led to a better diagnosis of the most probable disorder, as the difference between the prominent and the next one was bigger.

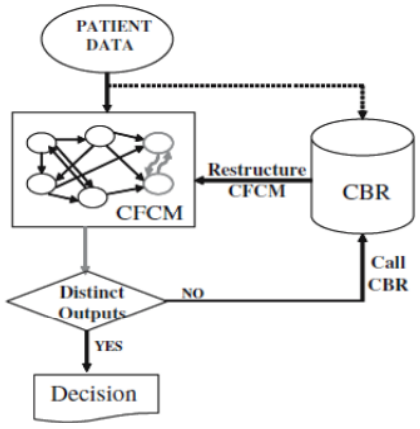

Fig. 5: Flowchart of CBR enhanced FCM algorithm for the advanced MDSS

The External Beam Radiotherapy Decision Support is another example that CBR-enhanced CFCMs contribute to the final choice for the optimum distribution of beam so as the healthy cells not be affected.

The integration of FCMs and case-based reasoning for medical decision support systems shows that in cases where CFCMs on their own do not provide clearly distinct decisions, the assistance from CBR leads to unambiguous decisions. CFCMs use historical data to infer a decision which make it less human dependant. However, the concept of time does not exist and this may be important in some cases leading to a different decision. In application such as radiotherapy, the volume of absorption may differ from organ to organ and the reaction time as well.

\subsubsection{CFCM complemented with Genetic Algorithm}

For the problems where the input information is not adequate and FCM-DSS cannot discriminate and reach a decision, a hybrid model was proposed [14]. Two complementary techniques have been used to overcome cases that a unique decision/diagnosis is not clear. It uses CFCM complemented with Genetic Algorithms Methods for concept interaction. GA offers an advance to the previous CFCMs as human intervention can be reduced.

CFCMs complemented with Genetic Algorithm take advantage the most essential characteristics of both techniques. Thus, the CFCM-GA is capable on its own to perform a comparison and lead to a decision based on expert opinions (structure of CFCM) using less human intervention, as Genetic algorithms are based on mimicking of the historical data and can compare and create new 'offspring' weights. Genetic Algorithms belong to adaptive methods exploiting existing data which are mainly used to solve search and optimization problems.

The GA component is used to "manipulate" weights that correspond to factor interactions and do not involve decision concepts, which can increase the complexity. Thus, in order to activate the GA component, it is formed the "reduced" weight matrix. The final decision corresponds to the decision concept with the highest 
probability resulted from the iterations. The detailed algorithm is described in [14].GAs can improve the quality of decision making.

In 2009, the Genetic Algorithm Factor Interaction Competitive FCM (GAFI-CFCM) Diagnostic Support Model has developed based on this hybrid model. It uses CFCMs as described in [11] and genetic algorithms [14]. Fig. 6 illustrates the overall MDSS Structure consisting of FCM and GA.

The GAFI-CFCM has been applied for differential diagnosis to speech and language pathology[14]. The results in comparison with those of CFCM proved that GAFI-CFCM gives a more 'clear' decision for the probable disorder.

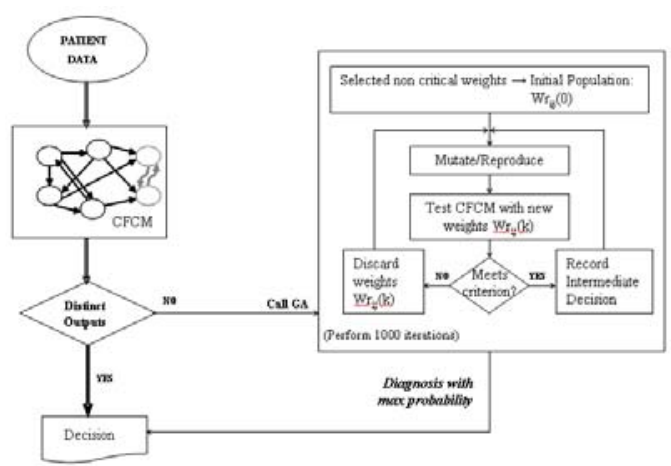

Fig. 6:Overall MDSS Structure consisting of FCM and GA

GAFI-CFCM has been also applied for labor modeling [15]. The results proved that this FCM-MDSS architecture, that uses CFCMs and GA, is a promising approach as it reaches the correct conclusion and infers the best decision.

\subsection{Distributed m-FCM}

For better handling cases of large complex systems, an approach is the decomposition into sub-systems. This technique has been used extensively on conventional approaches [16]. When subsystems have many elements in common, decomposition is not easy and that prohibit the simplified approach of summing up the individual components behavior.

The generic purpose of $\mathrm{m}$-FCMs is to receive information from all the subsystems in order to accomplish a task, make decisions and to plan strategically. The advantage of distributed m-FCM is that factors that correspond to a process can be organized to groups and each group constitutes a (sub)FCM, reducing the complexity. Each sub-FCM should be handled both as autonomous FCM system and as a part of a general interactive mFCM system.

For complex MDSS, every subsystem is modeled by a FCM and each component of the infrastructure is a part of the intricate web that forms the overall infrastructure [17] (Fig.7).

FCMs communicate with each other as they are in a common environment. The links denote the influence among the concepts. The connections create an intricate web, depending on the weights that characterize the links. Interdependencies among FCMs increase the overall complexity of the "system to systems".

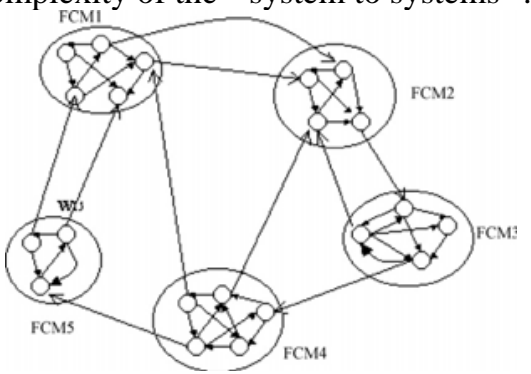

Fig.7: The distributed m-FCM model

The distributed m-FCMs have been applied for differential diagnosis of dysarthria and apraxia of speech, mainly for the differentiation among the types of dysarthria, since many speech and oral motor characteristics of the dysarthrias are overlapping. In the distributed mFCM some factors can be grouped together given that they represent separate assessment procedures, each group can be a FCM and a part of the distributed mFCM. Thus, in the distributed m-FCM model for the differential diagnosis system of dysarthria and apraxia of speech, the results of subsystem FCMs used for various assessments are aggregated into one combined distributed FCM. The comparison of diagnosis provided by a speech and language pathologist (SLP) and the dysarthria-apraxia distributed m-FCM gives the final decision as in some cases the type of dysarthria may not be so distinct (very close values). It can be combined with a supervisor gives the hierarchical structure, for better results.

\subsection{Hierarchical architecture}

The hierarchical architecture includes the advantages of the distributed m-FCMs and the existence of supervisor. Thus, the upper level -the supervisor- can perform some of the tasks that a human operator successfully performs in supervising systems. It can handle and express qualitative information and have knowledge about the process structure and determine on the acceptance of a result.

A hierarchical architecture is proposed where the mFCM can be used to model the supervisor. Fig. 8 illustrates a two-level hierarchical structure where m-FCM is used as supervisor. The m-FCM represents the relationships among the subsystems and their models while inferring the final decision by evaluating all the information from them [17].

FCMs have been used to model the supervisor for decision support actions during the labor[15]. The main decision of obstetricians is to determine whether they will proceed with a Caesarian section or a natural delivery based on the physical measurements, FHR and other essential indications and measurements.

The concepts of FCM are the main parameters that the obstetricians evaluate. Experienced ones have estimated the degree of influence from one concept to another and the FCM is constructed. The values of concepts are calculated according to the influence from interconnected concepts. At the lower level there are simple sensors or more advanced systems such as FHR 
classification system based on support vector machines. In the upper level is a CFCM as the concept of Caesarian section or not have to be mutually exclusive.

The hierarchical two-level architecture has been also used for decision making in radiation therapy [18]. Hierarchical architecture is used to make decisions about the final dose of beam for radiation therapy.

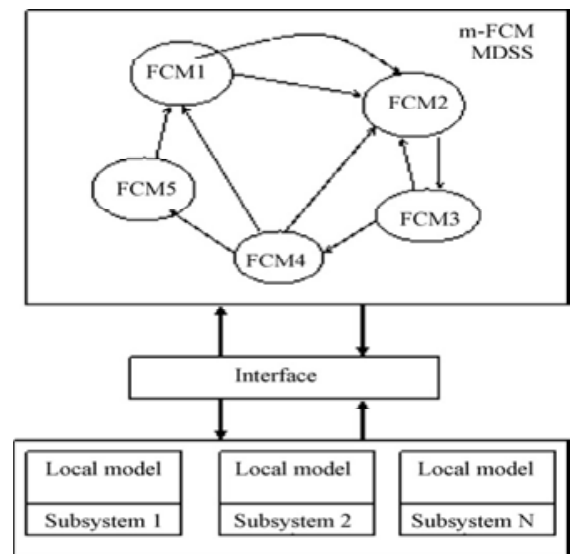

Fig. 8. The hierarchical architecture with the m-FCM for medical decision support systems

The concepts of the FCM model for radiotherapy treatment are divided into three categories: Factorconcepts, Selector-concepts, and Output-concepts. Input (factors and selectors) concepts, represent treatment variables with given or measured or desired values, and the corresponding causal weights are calculated. The final decision-making is based on the determination of the values of the Output-concepts that figure out the final decision.

The Supervisor-FCM evaluates the success or the failure of the treatment process by checking Final Dose value. This tool uses NHL in the supervisor-FCM, for combining the human experts' structural knowledge with the data for each specific case and constitutes an integrated hierarchical structure of Radiotherapy Decision Support System.

This structure is easily implemented in clinical practice and provides a fast, accurate, reliable, and flexible tool for decision-making in radiotherapy procedure.

\subsection{An optimization for the supervisor-FCM}

The weight matrix determines the causal-effect relationships among concepts. In a hierarchical structure, the supervisor-FCM enhances the simulation ability of the system, resulting in more reliable decision making. Thus, the optimal weight matrix for the FCM model is under constant survey.

Parsopoulos et. al. [19], proposed the use of Particle Swarm Optimization (PSO) and Differential Evolution (DE), coming from the fields of Swarm Intelligence and Evolutionary Computation respectively, to optimize the supervisor-FCM. PSO aims at learning FCM structure based on historical data that converge to the desire final state. The Differential Evolution is a method that optimizes a problem by iteratively trying to improve a candidate solution according to a given measure of quality.
The proposed method which includes PSO and DE algorithms led to the computation of optimum weight values by minimizing an objective function [19].

PSO is a population based algorithm, which performs a search for the solution by maintaining and transforming a population of individuals. The learning requires human knowledge that is used to specify adequate constraints, which would guarantee that the relationships within the FCM model retain the physical meaning defined by experts. However, this method minimizes the human intervention. PSO and DE are described in [20]. The proposed methods determine the cause-effect relationships among concepts, determining the optimal weight matrix for the supervisor-FCM model. Experiments showed that the optimum solution is achieved. However, this method used optimization only for the supervisor-FCM not for the lower level.

\subsection{Evolutionary-basedFCMs}

The need for reducing human intervention for constructing FCMs, as human experts are subjective, led to Evolutionary FCMs. For these FCMs, methods for automated generation of FCM models have developed. The proposed method is based on genetic algorithms [21] which can generate FCM models from historical data. The aim of the proposed learning method is to eliminate human intervention during development of a FCM model. This process is performed by exploiting information from historical data to compute FCM model connection matrix; hence it is able to mimic the data. It used with an extension of Genetic Algorithms the Real-Coded Genetic Algorithm (RCGA).The RCGA algorithm uses the input data to develop and optimize, with respect to the input data, connection matrix of a candidate FCM model.

The advantage of this FCM-model is that it can give a solution based on historical data, not on human factor, while it can be transparent, that is every node and edge within the FCM graph can be interpreted by a human. This model can 'predict' through learning FCM with long-term prediction capability [22]. It uses a learning method that based on the evolutionary algorithm,the Real Coded Genetic Algorithm.

RCGA algorithm has been used for prediction in medical cases such as the long-term prediction of prostate cancer. The used algorithm which is described in [22],is based on Real Coded Genetical Algorithm.

The first experimental analysis for the long-term prediction of prostate cancer justifies the advantage of using the proposed method. The produced evolutionarybased FCM, predicts the patient state after a period of time following a suggested therapy plan for the individual patient. The results compared to real medical data which can enhance the optimized results. However, it needs more experiments.

\section{Conclusions}

The area of MDSS is characterized by complexity requiring the investigation of new advanced methods for modeling and development of sophisticated systems. 
Soft computing techniques such as Fuzzy Cognitive Maps can be used for MDSS, achieving better solutions in diagnosis, treatment, prediction and so on. Some of them succeeded to give a distinct decision, while others needed enhancing in order to reach a clear decision. FCMs can be a powerful tool as the experience of many experts and knowledge from historical data can be combined and contribute to form the FCMs. These models can be useful and a valuable tool especially for inexperienced medical professionals. A comparison of the various FCM structures have been used for MDSS are gathered at Table 1.

In the future, the fact that FCMs are based on experts, with different background and experiences, makes them subjective. Thus, in order to have more reliable models corresponding to a real situation, a 'credibility’ parameter should be inserted. In addition, especially for medical problems, time may be important as it can influence in a different way the concepts and as a result the output. Therefore, temporal concept should be introduced. The construction of dynamic and more transparent structure is another significant parameter for medical problems.

\section{Acknowledgement}

This work was supported by the E.U. FP7-PEOPLEIAPP-2009, Grant Agreement No. 251589, Acronym: SAIL.

\section{References}

[1] Sprogar, M.; Lenic, M.; Alayon, S. Evolution in medical decision making. Journal of Medical Systems,36. 2002, pp. 479-89.

[2] Kosko, B. Fuzzy Cognitive Maps. International Journal of Man-Machine Studies. 1986, Vol. 24, pp. 65-75.

[3] Axelrod, R.Structure of Decision: the cognitive maps of political elites. NJ : Princeton, 1976.

[4] Degoulet, P. and Fieschi, M. Medical Decision Support Systems. Introduction to Clinical Informatics. New York: Springer , 1997, 12, pp. 153-167.

[5] Dickerson, J. and Kosko, B. Fuzzy Virtual Worlds. Artificial Intelligence Expert. 1994, Vol. 7, pp. 2531.

[6] Huerga, A. V. A Balanced Differential Learning algorithm in Fuzzy Cognitive Maps. In Proceedings of the 16th International Workshop on Qualitative Reasoning. 2002.

[7] Papageorgiou, E., Stylios, C and P., Groumpos. Fuzzy Cognitive Map Learning Based on Nonlinear Hebbian Rule. Australian Conf. on Artificial Intelligence. 2003, pp. 256-268.

[8] Papageorgiou, E., Stylios, C. D. and Groumpos, P. Active Hebbian Learning Algorithm to Train Fuzzy Cognitive Maps. J. Approx. Reason. 37 (3). 2004, pp. 219-249.

[9] Oja, E. Simplified neuron model as a principal component analyzer. Journal of Mathematical Biology. 1982, Vol. 16, pp. 267-273.
[10]Georgopoulos, V. C., Malandraki, G. A. and Stylios, C. D. A Fuzzy Cognitive Map Approach to Differential Diagnosis of Specific Language Impairment. Journal of Artificial Intelligence in Medicine. December 2003, pp. v.29, 221-278.

[11]Georgopoulos, V.C. and Stylios, C D. Augmented fuzzy cognitive maps supplemented with case based reasoning for advanced medical decision support. [ed.] M. Nikravesh, L. A Zadeh and J. Kacprzyk. Soft Computing for Information Processing and Analysis. Springer-Verlag, 2005, pp. 391-405.

[12]Georgopoulos, V. C. and Stylios, C. D. Complementary case-based reasoning and competitive fuzzy cognitive maps for advanced medical decisions. Soft Comput. 12(2). 2008, pp. 191-199.

[13]Krekelberg, B and Kok, J N. A lateral inhibition neural network that emulates a winner-takes-all algorithm. [ed.] M. Verleysen. in Proceedings of the European Symposium of Artificial Neural Networks. 1993, pp. 9-14.

[14]Georgopoulos, V.C. and Stylios, C.D. Diagnosis Support using Fuzzy Cognitive Maps combined with Genetic Algorithms. 31st IEEE Engineering in Medicine and Biology Society (EMBC'09). 2009.

[15]Stylios, C.D. and Georgopoulos, V.C. Fuzzy Cognitive Maps for Medical Decision Support - A Paradigm from Obstetrics. 32nd Annual International Conference of the IEEE EMBS Buenos Aires, Argentina. August 31 - September 42010.

[16]Mesarovic, M D, Macko, D and Takahara, Y.The Theory of Hierarchica IMultilevel Systems. New York : Academic Press, 1970.

[17]Stylios, C. D. The knowledge based technique of fuzzy cognitive maps for modeling complex systems,. in Proceedings of 16th European Meetingson Cybernetics and Systems Research (EMCSR). 2002, pp. 524-529.

[18]Papageorgiou, E. I., Stylios, C. D. and Groumpos, P. P. An Integrated Two-Level Hierarchical System forDecision Making in Radiation Therapy Based on Fuzzy Cognitive Maps. IEEE Transactions on Biomedical Engineering. December 2003, Vol. 50, 12.

[19]Parsopoulos, K E, et al. A First Study of Fuzzy Cognitive Maps Learning using Particle Swarm Optimization. IEEE 2003 Congr. on Evolutionary Computation. 2003, pp. 1440-1447.

[20]Parsopoulos, K. E., et al. Evolutionary Computation Techniques for Optimizing Fuzzy Cognitive Maps in RadiationTherapy Systems. Lecture Notes in Computer Science (LNCS). Spinger, 2004, Vol. 3102, pp. 402-413.

[21]Stach, W., et al. Evolutionary Development of Fuzzy Cognitive Maps. IEEE International Conference on Fuzzy Systems. 2005, pp. 619 -624.

[22]Froelich, W., et al.Application of evolutionary fuzzy cognitive maps to the long-term prediction of prostate cancer. Soft Computing. 2012, pp. 3810 3817. 
Table 1: FCM structures used for MDSS

\begin{tabular}{|c|c|c|c|c|}
\hline $\begin{array}{l}\text { Type of } \\
\text { FCM }\end{array}$ & $\begin{array}{l}\text { Type of De- } \\
\text { cision }\end{array}$ & $\begin{array}{l}\text { Main characteristics of the } \\
\text { approach/ MDSS }\end{array}$ & $\begin{array}{l}\text { Learning } \\
\text { Method }\end{array}$ & $\begin{array}{l}\text { Application } \\
\text { Field }\end{array}$ \\
\hline NHL-FCM & $\begin{array}{l}\text { Decision } \\
\text { mak- } \\
\text { ing/diagnosi } \\
\text { s, } \\
\text { classification }\end{array}$ & $\begin{array}{c}\text { Unsupervised learning; Synchronous triggering \& } \\
\text { interaction of concepts; One step approach; De- } \\
\text { mand human intervention; Problem-dependent; } \\
\text { Classification }\end{array}$ & $\begin{array}{l}\text { Non Line- } \\
\text { ar Hebbian } \\
\text { (NHL) }\end{array}$ & $\begin{array}{l}\text { Radiotherapy, } \\
\text { Tumor grading }\end{array}$ \\
\hline AHL-FCM & $\begin{array}{l}\text { Grade diag- } \\
\text { nostic deci- } \\
\quad \text { sion, } \\
\text { Classifica- } \\
\quad \text { tion }\end{array}$ & $\begin{array}{l}\text { Unsupervised learning; Asynchronous Weight up- } \\
\text { date; Asynchronous sequence of activation con- } \\
\text { cepts; Activation Decision Concepts (ADCs)- } \\
\text { desired outputs ; Demand human intervention } \\
\text { Problem-dependent; Classification }\end{array}$ & $\begin{array}{l}\text { Active } \\
\text { Hebbian } \\
\text { Learning } \\
\text { (AHL) }\end{array}$ & tumor grading \\
\hline CFCMs & $\begin{array}{l}\text { Differential } \\
\text { diagnosis, } \\
\text { critiquing }\end{array}$ & $\begin{array}{c}\text { Two main kinds of concepts: factors concepts \& } \\
\text { diagnosis concepts; One decision come up; } \\
\text { Factor concepts: inputs } \\
\text { Diagnosis concepts: outputs } \\
\text { One decision come up; Competiveness } \\
\text { Compare and lead to decision; decision based on } \\
\text { expert knowledge and experience }\end{array}$ & $\begin{array}{l}\text { Competi- } \\
\text { tive }\end{array}$ & $\begin{array}{l}\text { Specific Lan- } \\
\text { guage Impair- } \\
\text { ment }\end{array}$ \\
\hline $\begin{array}{l}\text { Augmented } \\
\text { CFCMs Sup- } \\
\text { plemented } \\
\text { with CBR }\end{array}$ & $\begin{array}{l}\text { Differential } \\
\text { diagnosis }\end{array}$ & $\begin{array}{l}\text { CFCM with CBR; Two main kinds of concepts: } \\
\text { factors concepts \& diagnosis concepts; One deci- } \\
\text { sion come up; CBR (store previous cases); Histori- } \\
\text { cal data; Not always CBR called; Nearest-neighbor } \\
\text { retrieval technique; Competiveness; Compare and } \\
\text { lead to decision; decision based on expert } \\
\text { knowledge and experience }\end{array}$ & $\begin{array}{l}\text { Competi- } \\
\text { tive }\end{array}$ & $\begin{array}{l}\text { Specific Lan- } \\
\text { guage Impair- } \\
\text { ment }\end{array}$ \\
\hline $\begin{array}{l}\text { Complemen- } \\
\text { tary CFCM - } \\
\text { CBR en- } \\
\text { hanced }\end{array}$ & $\begin{array}{l}\text { Differential } \\
\text { diagnosis , } \\
\text { Decision } \\
\text { making }\end{array}$ & $\begin{array}{l}\text { CFCM with CBR; Critical factor concepts; Lateral } \\
\text { inhibition; Double check diagnosis- concept; Less } \\
\text { search space and time; Reduction in simulation } \\
\text { time; Simulation time reduction; Compare and lead } \\
\text { to decision; decision based on expert knowledge } \\
\text { and experience and tested previous cases }\end{array}$ & $\begin{array}{l}\text { Competi- } \\
\text { tive }\end{array}$ & $\begin{array}{l}\text { Specific Lan- } \\
\text { guage Impair- } \\
\text { ment, } \\
\text { Radiotherapy }\end{array}$ \\
\hline $\begin{array}{l}\text { CFCM com- } \\
\text { plemented } \\
\text { with Genetic } \\
\text { Algorithm }\end{array}$ & $\begin{array}{l}\text { Differential } \\
\text { diagnosis, } \\
\text { Decision } \\
\text { making, } \\
\text { modeling }\end{array}$ & $\begin{array}{l}\text { CFCM structure; Two main kinds of concepts: } \\
\text { factors concepts \& diagnosis concepts; Not always } \\
\text { GA called; GA ‘manipulate’ weight matrix; Re- } \\
\text { duced weight matrix; Less human intervention; } \\
\text { Compare and lead to decision }\end{array}$ & $\begin{array}{l}\text { Competi- } \\
\text { tive Ge- } \\
\text { netic Al- } \\
\text { gorithm }\end{array}$ & $\begin{array}{l}\text { Speech and } \\
\text { language } \\
\text { pathology, } \\
\text { Labor } \\
\text { modeling }\end{array}$ \\
\hline $\begin{array}{l}\text { Distributed } \\
\text { m-FCMs }\end{array}$ & $\begin{array}{l}\text { Differential } \\
\text { diagnosis }\end{array}$ & $\begin{array}{l}\text { Subsystems; FCM for each subsystem; Grouped } \\
\text { factors; Communication among FCMs; Intricate } \\
\text { web }\end{array}$ & - & $\begin{array}{l}\text { Speech and } \\
\text { language } \\
\text { pathology }\end{array}$ \\
\hline $\begin{array}{l}\text { Supervisor } \\
\text { FCM }\end{array}$ & $\begin{array}{l}\text { Decision } \\
\text { making, } \\
\text { Treatment }\end{array}$ & $\begin{array}{c}\text { Uses Distributed m-FCMs; Determine the treatment } \\
\text { acceptance or not; Enhance simulation ability; Evo- } \\
\text { lutionary algorithms for optimization; Less human } \\
\text { intervention }\end{array}$ & $\begin{array}{l}\text { NHL, } \\
\text { PSO } \\
\&(\mathrm{DE})\end{array}$ & $\begin{array}{l}\text { Labor, } \\
\text { Radiotherapy }\end{array}$ \\
\hline $\begin{array}{l}\text { Hierarchical } \\
\text { (m-FCM } \\
\text { MDSS\& } \\
\text { Supervisor) }\end{array}$ & $\begin{array}{l}\text { Decision } \\
\text { making, } \\
\text { Treatment }\end{array}$ & $\begin{array}{l}\text { Supervisor FCM; Three kinds of concepts: Factor- } \\
\text { concepts, Selector- concepts, and Output- con- } \\
\text { cepts; Factor \& Selector concepts (inputs);Control } \\
\text { by the supervisor }\end{array}$ & $\begin{array}{l}\text { mFCM: - } \\
\text { Supervisor } \\
\text { FCM: } \\
\text { NHL,PSO } \\
\text { \&(DE) }\end{array}$ & $\begin{array}{l}\text { Labor, } \\
\text { Radiotherapy }\end{array}$ \\
\hline $\begin{array}{l}\text { Evolutionary } \\
\text { based FCMs }\end{array}$ & $\begin{array}{l}\text { Clinical } \\
\text { guidelines, } \\
\text { prediction }\end{array}$ & $\begin{array}{l}\text { Experts; Eliminate human intervention; FCM from } \\
\text { historical data; Optimization Candidate FCM; }\end{array}$ & $\begin{array}{l}\text { Real } \\
\text { Coded } \\
\text { Genetic } \\
\text { Algorithm }\end{array}$ & $\begin{array}{l}\text { Long-term } \\
\text { prediction of } \\
\text { prostate cancer }\end{array}$ \\
\hline
\end{tabular}

